\title{
Results of the Computerized Mental Health Screening System for Female Offenders
}

\author{
Lynn A Stewart*, Kyle Archambault and Geoffrey Wilton
}

Correctional Service of Canada, Canada

\begin{abstract}
Objective: To estimate the proportion of incoming female offenders in the Canadian federal correctional system requiring mental health services based on results of a Computerized Mental Health Intake Screening System (COMHISS).

Method: Consecutive admissions to the five regional women's prisons in the Correctional Service of Canada were approached to consent to participate in the CoMHISS. The screening process combines two psychological self-report measures, the Brief Symptom Inventory, and the Depression Hopelessness and Suicide Screening Form. Results were analyzed based on the percentage of women who met established cut-off scores on the measures and further analyzed by Aboriginal ethnicity.

Results: Sixty-two percent of the sample reported elevated levels of psychological distress that would warrant further assessment. Although higher for Aboriginal women, the mean scores did not differ significantly from those of non-Aboriginal women. The rate of co-occurring substance abuse among women reporting psychological distress was estimated at $70 \%$.

Conclusions: Planning for the delivery of mental health services for federally sentenced women should consider their high rates and variety of psychological problems. Specific correctional treatment planning requires attention to criminogenic needs as well as mental health issues and serious substance abuse problems. Mental health providers for women in prison should be aware of the significant likelihood of co-morbid substance abuse disorders and mental health problems and prepare women to identify follow up services to address these problems on release.
\end{abstract}

Keywords: Female offender; Mental health screening; Brief symptom inventory; Depression hopelessness suicide form; Prevalence; Psychological problems

\section{Introduction}

Addressing the needs of female offenders with mental disorders is an increasing concern for correctional agencies. There are higher rates of mental disorder among female offenders than among women in the non-offender population [1-5] and higher rates among female offenders than male offenders [6-9]. These findings are consistent with trends in the Correctional Service of Canada (CSC) where the proportion of incoming women who report histories of mental disorder has increased from $20 \%$ to $31 \%$ over a ten year period from March 1997 to March 2008 [10]

There are fewer studies providing estimates of the prevalence of mental disorder involving female offenders than male offenders. Different methodologies (particularly variation in sample selection, measurement tools, timeframes, and definitions of mental disorder) have produced rates as high as $84 \%$ when substance abuse disorders are included [5] and 93\% in one Iowa prison when life time incidence criteria are applied [11]. Aboriginal female offenders may be more likely than non-Aboriginal women to have higher rates of psychological distress and to positively screen for symptoms of psychosis and affective disorders [2] Female offenders also have elevated rates of co-occurring mental and substance abuse disorders $[12,13]$.

In CSC, offenders' current and past mental health status had previously been assessed by questions posed to them on entry into the federal correctional system as part of their intake assessment. The information collected by these questions was sufficient to monitor general trends, but were not an adequate basis for determining the need for further assessment or treatment. Evidence also suggests that these types of questions may underestimate the number of inmates with serious mental disorders [14]. The adoption of a standardized mental health screening tool in CSC would assist in identifying offenders who require further assessment and in establishing an immediate triage system for offenders at risk for self-injury. As part of a comprehensive strategy to address the offenders' mental health needs, CSC developed and implemented the Computerized Mental Health Intake Screening System (CoMHISS) in 2009. Screening for mental health problems through CoMHISS is the initial component of the continuity of care established for federally sentenced offenders with mental health needs. Those inmates who are assessed as meeting a specified cutoff score on the mental health screening instruments are referred for further assessment by a mental health professional. The assessment information allows staff to prescribe the type and level of mental health intervention required to meet offenders' needs. These data will allow administrators to monitor changes in the prevalence rates of inmates' self-reported mental health problems and target resources for regions and institutions with higher numbers of offenders with these problems.

Female offenders in CSC have been sentenced by the courts to a term of two or more years. Relative to provincial offenders, they tend to be serving sentences for the more serious crimes. Currently, there are over 900 women serving a federal sentence in Canada. This paper presents

*Corresponding author: Lynn A Stewart, Senior Research Manager, Correctional Service of Canada, Canada, Tel, 416-804-3223; E-mail: StewartLA@csc-scc.gc.ca

Received July 03, 2013; Accepted July 23, 2013; Published July 31, 2013

Citation: Stewart LA, Archambault K, Wilton G (2013) Results of the Computerized Mental Health Screening System for Female Offenders. J Socialomics 2: 103. doi:10.4172/2167-0358.1000103

Copyright: (C) 2013 Stewart LA, et al. This is an open-access article distributed under the terms of the Creative Commons Attribution License, which permits unrestricted use, distribution, and reproduction in any medium, provided the original author and source are credited. 
the results of the national pilot of the CoMHISS for incoming federally sentenced women. It focuses on the estimation of the proportion of female inmates experiencing psychological distress based on specific cut-off scores on two self-report psychological inventories.

\section{Method}

\section{Measures/material}

Three measures were chosen as the components of the initial version of CoMHISS: the Paulhus Deception Scale (PDS), and two psychological screening tools, the Brief Symptom Inventory (BSI) [15] and the Depression, Hopelessness and Suicide Screening Form (DHS) [16]. The latter two instruments were chosen based their capacity to identify individuals experiencing high levels of psychological distress. The PDS [17] was chosen to determine to what extent the scores on the screening tools might be affected by the respondents' impression management.The summary of the measures that comprise the CoMHISS presented below is reproduced from the initial report on the results of the pilot of the CoMHISS report for men [18].

\section{The Depression, Hopelessness and Suicide Screening Form (DHS)}

The DHS form measures two constructs: depression (17 items) and hopelessness (10 items), in addition to screening for risk factors associated with suicide/self-harm (12 items). Elevations on the Depression scale indicate endorsement of thoughts and feelings of sadness, disappointment and failure. Items reflecting difficulties sleeping, lack of energy and loss of appetite are included in the scale. High scores on the Hopelessness scale suggest the respondent sees the future as bleak and unhappy. A third component of the measure is the 12 items associated with risk for suicide; among these are three items that assess current suicide ideation. The DHS has been found to have acceptable psychometric properties when applied to federally sentenced female offenders in Canada $[19,20]$ where research has demonstrated that the DHS was generally equivalent to interviews and file reviews at identifying inmates with a history of self-harm.

\section{Scoring, interpretation and recommended cut-off scores}

The authors of the DHS have advised that T-scores can be used to determine suitable cut-offs for a given population and have established specific normative interpretative ranges for the Depression and Hopelessness scales for women [20]. Because the CoMHISS is administered at intake, the intake norms on the DHS were used. For this study, female offenders with T-scores of 65 and above and T-scores of 60 and above were identified. The measure's authors advise mental health professionals to establish their own decision rules around interventions for respondents endorsing the suicide risk items, but they recommend that endorsement of any one of the three items related to current suicidal ideation warrants a further assessment for potential risk for suicide.

\section{The Brief Symptom Inventory (BSI)}

The BSI [15] is a 53 item self report symptom inventory that assesses nine patterns of clinically relevant psychological symptoms. It is a brief version of the Symptom Checklist 90-Revised (SCL-90-R). Correlations between the BSI and SCL-R-90 are reported to range from .92 to .99 [15]. The BSI has been used in a variety of clinical and counselling settings as a mental health screening tool and as a method of measuring symptom reduction during and after treatment. It has been demonstrated to retain its reliability and validity in cross cultural samples [21]. The nine primary symptom dimensions the scale measures are: Somatization (distress arising from perceptions of bodily dysfunction), Obsession-Compulsion (thoughts and impulses that are experienced as unremitting and irresistible but are of an unwanted nature), Interpersonal Sensitivity (feelings of personal inadequacy and inferiority in comparison with others), Depression (symptoms of dysphoric mood and affect as well as lack of motivation and loss of interest in life), Anxiety (nervousness and tension as well as panic attacks and feelings of terror), Hostility (thoughts, feelings or actions that are characteristic of anger), Phobic anxiety (persistent fear response to a specific place, object or situation that is irrational), Paranoid ideation (disordered thinking characteristic of projective thoughts, hostility, suspiciousness, grandiosity, fear of loss of autonomy, and delusions) and Psychoticism (withdrawn, isolated, schizoid lifestyle as well as first rank symptoms of schizophrenia such as thought control). The BSI also includes three indices of global distress. The Global Severity Index (GSI), Positive Symptom Distress Index, and Positive Symptom Total measure current level of symptomology, intensity of symptoms, and number of reported symptoms, respectively.

\section{Scoring, interpretation and recommended cut-offs}

Raw scores on the nine primary symptom dimensions and the GSI are calculated by summing the values for the items in each dimension (including four additional items for the GSI) and then dividing by the number of endorsed items in that dimension. The Positive Symptom Total is calculated based on the total count of the number of non-zero responses and reveals the number of symptoms the respondent reports experiencing. The Positive Symptom Distress Index is calculated by summing the values of the items receiving non-zero responses divided by the Positive Symptom Total. This index provides information about the average level of distress the respondent experiences. T-scores are calculated by comparing a given raw score to the normative tables from a given population. Frequently, a cut-off T-score of 65 is used in identifying very high scores. Only about $7 \%$ of the referent population would score at this level or higher.

The BSI manual provides norms based on four groups each separated for males and females: adult non patients, adult psychiatric outpatients, adult psychiatric inpatients and adolescent non patients. The test author advises that the Global Severity Index is the scale that is the most sensitive single indicator of distress. The author advises that using the reference group of adult non-patients, a T-score of 63 or above on the GSI or a T-score of 63 and above on any two dimensions would be considered a case worthy of further evaluation [15]. This study examined the percentage of female offenders who fell at or above T-scores of 60 and 65 in order to determine the number of cases that would be flagged for follow-up using these criteria. The adult female non-patient group was chosen as the reference population.

\section{Profile information}

Profile information on the CoMHISS participants was extracted from a comprehensive offender intake assessment conducted on all offenders in CSC. The Dynamic Factors Identification and Analysis (DFIA) component assesses dynamic risk factors or criminogenic needs grouped into seven domains. The DFIA yields ratings of need levels for each domain, as well as an overall level of dynamic need ranging from low to high. The principle tool used for assessing risk level of female offenders is the Static Factors Assessment which provides comprehensive information pertaining to the criminal history and risk factors yielding an overall level of low, medium, or high static risk. Both measures have been found to be reliably related 
Citation: Stewart LA, Archambault K, Wilton G (2013) Results of the Computerized Mental Health Screening System for Female Offenders. J Socialomics 2: 103. doi:10.4172/2167-0358.1000103

Page 3 of 5

to correctional outcomes [22]. In addition, a computerized substance abuse assessment evaluates the extent of substance misuse and its relationship to offending is also conducted at intake. This includes the results of several well validated measures of substance misuse including the 20-item Drug Abuse Screening Test (DAST) [23] and the Alcohol Dependency Scale (ADS) [24,25].

\section{Procedure}

Staff from the psychology department of the reception units of women's facilities in CSC administered the questionnaires to all consecutive incoming participants who provided informed consent. Results were entered into a central database. The analyses include descriptive statistics on the scores of participants on the three measures in the CoMHISS assessment as well as background information on the CoMHISS participants and the incoming female offenders who entered CSC at the same time as the participants but did not receive the CoMHISS assessment. In addition, inmates who successfully completed the CoMHISS were compared to a small group of inmates who refused the assessment to determine if there was evidence of a selection bias. Chi-square analyses were conducted to determine if differences between groups were significant.

\section{Participants}

Female offenders entering one of the five regional institutions for women on a new sentence from January 2009 to June 2010 period were asked to participate and complete the measures after signing a consent form $(N=306)$. Of these, 41 refused and one offender's results were unavailable to the research team resulting in a sample of 264 participants. One participant had no results on the DHS, but was included in the analyses for the other measures. None of the women who participated produced results that were invalid.

During the year 2009-2010, CSC admitted 502 female offenders on new sentences. The participation of some of the institutions in the CoMHISS pilot began later in the study period. To ensure that the women who participated in the CoMHISS were representative of the total population of new inmates, the demographic profiles of CoMHISS respondents were compared to those who came into custody at the same time on new sentences, but who did not participate in the pilot. There was no difference in the proportion of Aboriginal inmates indicating that no selection bias in the participation of Aboriginal respondents in the CoMHISS, and no differences in current offence, sentence length, substance abuse problems or risk level. A weak significant difference was observed between the two groups on summary need level $\left(\chi^{2}(2)=7.26\right.$,
$P=.03$, Cramer's $\varphi=.14$ ). Overall, the results suggest that there was no selection bias in the recruitment of CoMHISS participants.

Forty-one (13.4\%) female offenders declined to participate in the CoMHISS out of 306 who were approached. Comparing the women who declined to those who accepted determined that they were significantly more likely to be Aboriginal $\left(\chi^{2}(1)=15.53, P<0.001\right.$, Cramer's $\varphi=.25$ ), but there were no differences with respect to risk level, need level, or major offence.

\section{Results}

\section{Results of the CoMHISS assessment}

The overall mean scores and two groupings of T-scores for the nine primary symptom dimensions and the three global indices of psychological distress in the BSI are reported in Table 1. The focus is placed on the GSI results since the measure's author recommends it is the most sensitive single indicator of respondents' psychological distress [15]. The GSI shows that over $45 \%$ of incoming female offenders score at T65 or above. The more liberal cut-off score of T60 would increase this estimate to over $65 \%$.

Comparisons conducted between Aboriginal and non-Aboriginal female offenders found no difference between the two groups' mean raw scores on the GSI or in the proportion of offenders screening in on the GSI. Furthermore, the difference between the frequency of Aboriginal and non-Aboriginal female offenders scoring above the T65 criterion across the nine primary symptom dimensions was not statistically significant. Aboriginal and non-Aboriginal female offenders tend to score similarly on the BSI in terms of the types of symptoms that are most problematic.

The DHS measure has been validated for federally sentenced women and specific norms established. Table 2 presents the results for the women who participated in CoMHISS using the specific intake norms for the Depression and Hopelessness scales. The measure's authors recommend that endorsement of one indicator on the current suicide ideation scale would warrant further assessment [20]. Just over $7 \%$ of the participants endorsed one or more of the current suicidal ideation critical items and about $37 \%$ endorse at least one item on the historical suicide indicator scale.

Differences between Aboriginal and non-Aboriginal women's results on the DHS indicated that Aboriginal offenders endorsed more symptoms of depression and hopelessness, but the differences were not statistically significant. Likewise, the percentage of Aboriginal and nonAboriginal women who endorsed at least one current suicidal ideation

\begin{tabular}{|c|c|c|c|}
\hline \multirow{2}{*}{ Dimension or Index } & \multirow{2}{*}{ Mean Raw Scale Score $N=264$} & T-score $\geq 60$ & T-score $\geq 65$ \\
\hline & & $\%(n)$ & $\%(n)$ \\
\hline Somatization & 0.86 & $47.4(125)$ & $34.5(91)$ \\
\hline Obsessive-Compulsive & 1.35 & $57.6(152)$ & $48.5(128)$ \\
\hline Interpersonal Sensitivity & 1.15 & $51.9(137)$ & $35.2(93)$ \\
\hline Depression & 1.22 & $67.0(177)$ & $46.6(123)$ \\
\hline Anxiety & 1.12 & $54.5(144)$ & $37.5(99)$ \\
\hline Hostility & 0.63 & $29.5(78)$ & $18.6(49)$ \\
\hline Phobic Anxiety & 0.57 & $37.9(100)$ & $24.6(65)$ \\
\hline Paranoid Ideation & 1.25 & $59.5(157)$ & $49.6(131)$ \\
\hline Psychoticism & 1.15 & $77.7(205)$ & $60.2(159)$ \\
\hline Global Severity Index (GSI) & 1.06 & $65.5(173)$ & $45.8(121)$ \\
\hline Positive Symptom Total (PST) & 26.69 & $61.4(162)$ & $39.0(103)$ \\
\hline Positive Symptom Distress Index (PSDI) & 1.89 & $58.0(153)$ & $43.2(114)$ \\
\hline
\end{tabular}

Table 1: BSI Results: T-Scores Based on Non Patient Norms. 
Citation: Stewart LA, Archambault K, Wilton G (2013) Results of the Computerized Mental Health Screening System for Female Offenders. J Socialomics 2: 103. doi:10.4172/2167-0358.1000103

\begin{tabular}{|c|c|c|c|}
\hline \multirow{2}{*}{ DHS Scale } & \multirow{2}{*}{ Mean } & T-score $\geq 60$ & T-score $\geq 65$ \\
\hline & & $\%(n)$ & $\%(n)$ \\
\hline Depression & 7.42 & $21.7(57)$ & $15.2(40)$ \\
\hline Hopelessness & 2.14 & $16.0(42)$ & $14.1(37)$ \\
\hline & & \multicolumn{2}{|c|}{ Suicide Items } \\
\hline & & \multicolumn{2}{|c|}{ Endorses $\geq 1$ item $\%(n)$} \\
\hline Cognitive Suicide Indicator & 0.09 & \multicolumn{2}{|c|}{$8.4(22)$} \\
\hline Current Ideation Indicator & 0.08 & \multicolumn{2}{|c|}{$7.2(19)$} \\
\hline Historical Suicide Indicator & 0.99 & \multicolumn{2}{|c|}{$37.3(98)$} \\
\hline
\end{tabular}

Table 2: DHS Results: T-scores Based on Women Offender Norms $(N=263)$

item was not significantly different. However, there is a significant difference in their self-reported histories of suicide ideation and selfinjury. Fifty-eight percent of Aboriginal offenders report a prior history of suicidal ideation, self-harm and suicide attempts compared to $30.2 \%$ of non-Aboriginal women $\left(\chi^{2}(1)=11.61, P<0.001\right.$, Cramer's $\left.\varphi=.23\right)$.

The PDS assesses the validity of an individual's responses to selfreport measures. The results indicated that fewer than $4 \%$ of female offenders had T70 scores on the IM scale. This indicates that very few of the participants were consciously presenting themselves falsely. Over $20 \%$ of offenders had scores at T70 or above on the SDE scale meaning that their responses were likely to be mediated by an unconscious tendency to give honest but exaggerated responses. There were no significant differences in these results for Aboriginal or non-Aboriginal women.

\section{Additional screening criteria}

Applying recommended screening criteria on all the scales further increases the proportion of offenders who would be screened in. The complete screening criteria include: 1) a GSI T-score greater than or equal to 65,2$)$ two or more BSI primary symptom dimensions with T-scores greater than or equal to 65,3 ) a DHS Depression scale score greater than or equal to 14,4 ) a DHS Hopelessness scale score greater than or equal to 6 , and 5) one or more endorsed items on the current suicide ideation scale on the DHS. Sixty-two percent of the sample of federally sentenced women would be screened in for further evaluation using all the criteria. Examined by Aboriginal status, results indicate that $59 \%$ of non-Aboriginal and $68 \%$ of Aboriginal women inmates would require additional assessment.

Future research is required to assist in identifying the optimal screening criteria given correctional priorities. More conservative criteria create more false negative cases, resulting in the screening possibly missing identifying offenders who may need mental health services. While more liberal criteria generate fewer false negative cases, they are subject to more false positive cases, which can place considerable burden on mental health teams that have to service offenders, some of whom may not actually require treatment. Regardless of the final criteria selected, these results show that a significant proportion of the incoming federally sentenced women population will require at least further follow-up assessment.

\section{Co-occurring disorders}

The literature has documented high rates of co-occurring mental health and substance abuse problems among female offenders $[5,26,27]$. None of the measures on the CoMHISS assess substance abuse. However, several measures included in the offender intake assessment provide information on the extent of offenders' drug and alcohol use and their level of dependence. Research has established that moderate and higher on the ADS and DAST are closely related to DSM-IV diagnoses of substance abuse problems [28]. In this sample,
$70 \%$ of female offenders with elevated scores on the CoMHISS also had moderate to severe scores on the ADS or the DAST. Women who are screened-in for further services based on the CoMHISS are significantly more likely to have significant substance abuse problems than women who are not screened-in $\left(\chi^{2}(1)=5.65, P=.017\right.$, Cramer's $\left.\varphi=.16\right)$.

\section{Discussion}

Based on the results of the Global Severity Index of the Brief Symptom Inventory applying a T 65 cut-off score, nearly $46 \%$ of federally sentenced women report elevated levels of psychological distress. These are very high rates relative to those expected in the normative population of women cited in the BSI manual $(\chi 2(1)=649.2, P<0.001$, Cramer's $\varphi=1.57)$ and higher than those recently found among male federal offenders, where $37.6 \%$ were screened-in for further assessment based on the same measure and criteria [18]. Aboriginal and non-Aboriginal female offenders report similar levels of distress on the CoMHISS, suggesting that there may be no need to develop separate norms for Aboriginal women. Using the full criteria, including the BSI dimension scores and all the elements of the DHS, over $60 \%$ of incoming women would need further assessment, and possibly, further mental health services. These figures are consistent with the very high rates of mental disorder recently found among a representative sample of federal women, $69 \%$ of whom met the DSM-IV diagnosis lifetime diagnostic criteria for major depressive episodes, $83 \%$ for antisocial personality disorder, and $80 \%$ for substance abuse disorder [29]. In addition, $70 \%$ of women in CSC scoring above the established cut-off scores on the CoMHISS have serious problems with substance abuse. This rate of cooccurring mental health and substance abuse problems is much higher than estimates from the general Canadian population [30,31] although the researchers have also noted the same pattern of criminal justice involvement, substance use problems and mental health issues among subpopulations in their sample. Reviews of the clinical literature have found that, unlike men, women's psychological problems typically precede their serious alcohol abuse [32]. This suggests that, for women, treating their mental health issues may facilitate reduction in substance abuse. The impact of first addressing substance abuse or psychological problems, or of addressing them in tandem, on treatment outcome, has not been well examined for this correctional population.

CoMHISS is meant to be administered within the first two weeks on admission to the institution. Admission to a federal institute is likely to be stressful and, therefore, one might expect, at least temporarily, elevated scores on the CoMHISS. Previous research [18] found that the scores on the DHS for women completing the measure at intake were higher relative to those who completed it later in their sentence. In the current study, over $75 \%$ of the women were tested in the first two weeks of entering CSC making an assessment of change in scores over time difficult. However, we confirmed that scores for the few women tested towards the end of their ninety day reception period did not differ from those tested earlier. Nevertheless, we acknowledge that rates of distress may be dynamic depending on the offenders' circumstances during their sentence.

\section{Conclusions}

Future research is required that would validate the CoMHISS screening measures and various cut-off scores against clinical diagnostic findings and female offenders' behaviour and psychiatric status while incarcerated. Such research would provide CSC with important information on the relationship of initial measures of psychological distress to correctional and mental health outcomes. Further research should also examine the extent to which correctional planning and 
Citation: Stewart LA, Archambault K, Wilton G (2013) Results of the Computerized Mental Health Screening System for Female Offenders. J Socialomics 2: 103. doi:10.4172/2167-0358.1000103

Page 5 of 5

mental health treatment for female offenders are complicated by the challenge of co-occurring psychological problems and serious alcohol or drug abuse. CoMHISS results provide a prevalence rate of federally sentenced female offenders who self-report a level of psychological distress, but estimates of the prevalence of mental disorder are best established by the use of standardised diagnostic tools by qualified practitioners. Based on recent research, it appears that the rate of mental disorder among federally sentenced females may be high enough that triage with a mental health screening system would not be warranted, and that all incoming women should be reviewed for level and type of mental health service required.

\section{Acknowledgements}

This study was funded through the support of the Correctional Service of Canada, Research Branch. The authors would like to thank the following for their contribution to the project and their thoughtful comments on initial drafts of the report: Colette Cousineau, Steve Varrette, Brian Grant and Michael Martin.

\section{Disclaimer}

The views and opinions expressed in this report are those of the authors and should not be construed to represent the views of any of the Correctional Service of Canada or the Canadian government.

\section{Disclosure Statement}

The authors have no actual or potential conflict of interest related to this research.

\section{References}

1. Blanchette K, Motiuk LL (2005) Female offenders with and without major mental health problems: a comparative investigation. Correctional Service of Canada.

2. Butler T, Allnutt $S$, Kariminia $A$ (2007) Mental health status of Aboriginal and non-Aboriginal Australian prisoners. Aust N Z J Psychiatry 41: 429-435.

3. Diamond PM, Wang EW, Holzer CE (2001) The prevalence of mental illness in prison. Adm Policy Ment Health 29: 21-40.

4. Scott CL (2010) Handbook of correctional mental health. (2nd edition). American Psychiatric Publishing Inc., London.

5. Tye CS, Mullen PE (2006) Mental disorders in female prisoners. Aust N Z J Psychiatry 40: 266-271

6. Fazel S, Danesh J (2002) Serious mental disorder in 23,000 prisoners: a systematic review of 62 surveys. Lancet 359: 545-550.

7. Ogloff JRP (2002) Identifying and accommodating the needs of mentally ill people in gaols and prisons. Psychiatr Psychol Law 9: 1-33.

8. Palmer EJ, Jinks M, Hatcher RM (2003) Substance use, mental health, and relationships: a comparison of male and female offenders serving community sentences. Int J Law Psychiatry 33: 89-93.

9. Steadman HJ, Osher FC, Robbins PC (2009) Prevalence of serious mental illness among jail inmates. Psychiatr Serv 60: 761-765.

10. Correctional Service of Canada (2008) Unpublished raw data. Correctional Service of Canada Offender Management System.

11. Gunter TD, Arndt S, Wenman G (2008) Frequency of mental and addictive disorders among 320 men and women entering the lowa prison system: use of the MINI-Plus. J Am Acad Psychiatry Law 36: 27-34.

12. Salina DD, Lesondak LM, Razzano LA (2007) Co-occurring mental disorders among incarcerated women: preliminary findings from an integrated health treatment study. Ment Health Issues Crim Justice Syst 45: 207-225.

13. Teplin LA, Abram KM, McClelland GM (1996) Prevalence of psychiatric disorders among incarcerated women. Arch Gen Psychiatry 53: 505-512.

14. Teplin LA (1990) The prevalence of severe mental disorder among male urban jail detainees: comparison with the Epidemiologic Catchment Area Program. Am J Public Health 80: 663-669.

15. Derogatis LR (1993) BSI Brief Symptom Inventory: administration, scoring, and procedures manual. (4th Ed.). National Computer Systems, Minneapolis.

16. Mills JF, Kroner DG (2004) A new instrument to screen for depression, hopelessness and suicide in offenders. Psychol Serv 1: 83-91.

17. Paulhus DL (1999) Paulhus Deception Scale. Multi-Health Systems Inc. Toronto.

18. Stewart LA, Harris A, Wilton G (2010) An initial report on the results of the pilot of the Computerized Mental Health Intake Screening System (CoMHISS). Correctional Service Canada.

19. Kroner DG, Kang T, Mills JF, Harris AJA, Green MM (2011) Reliabilities validities, and cutoff scores of the Depression Hopelessness Suicide Screening Form among women offenders. Crim Justice Behav 38: 779-795.

20. Mills JF, Kroner DG (2005) Screening for suicide risk factors in prison inmates: evaluating the efficiency of the Depression, Hopelessness, and Suicide Screening Form (DHS). Legal Crim Psychol 10: 1-12.

21. Aroian KJ, Patsdaughter C, Levin A (1995) Use of the Brief Symptom Inventory to assess psychological distress in three immigrant groups. Int J Soc Psychiatry 41: $31-46$

22. Motiuk LL (1997) Classification for correctional programming: the Offende Intake Assessment (OIA) process. Forum on Corrections Research 9: 18-22.

23. Skinner HA, Horn JL (1984) Alcohol Dependence Scale (ADS). Addiction Research Foundation, Toronto.

24. Skinner HA (1982) The Drug Abuse Screening Test. Addict Behav 7: 363-371.

25. Abram KM, Teplin LA, McClelland GM (2003) Comorbidity of severe psychiatric disorders and substance use disorders among women in jail. Am J Psychiatry 160: 1007-1010.

26. Sacks JY (2004) Women with co-occurring substance use and mental disorders (COD) in the criminal justice system. Behav Sci Law 22: 449-466.

27. Shinkfield AJ, Graffam J, Meneilly S (2009) Co-morbidity of conditions among prisoners. J Offender Rehabil 48: 350-365.

28. Peters RH, Greenbaum PE, Steinberg ML (2000) Effectiveness of screening instruments in detecting substance use disorders among prisoners. J Subst Abuse Treat 8: 349-358.

29. Derkzen D, Booth L, Taylor K, McConnelle A (2013) Mental health needs of federal female offenders. Psychol Serv 10: 24-36.

30. Rush B, Koegl CJ (2008) Prevalence and profile of people with co-occurring mental and substance use disorders within a comprehensive mental health system. Can J Psychiatry 53: 810-821.

31. Rush B, Urbanoski K, Bassani D (2008) Prevalence of co-occurring substance use and other mental disorders in the Canadian population. Can J Psychiatry 53: 800-809.

32. Zilberman ML, Tavares H, Blume SB (2003) Substance use disorders: sex differences and psychiatric comorbidities. Can J Psychiatry 48: 5-13. 